\title{
Gut Microbial Dysbiosis is Correlated with Stroke Severity Markers in Aged Rats Following Stroke
}

Tyler C. Hammond

University of Kentucky College of Medicine

\section{Sarah Messmer}

University of Kentucky College of Medicine

Jaqueline A. Frank

University of Kentucky College of Medicine

Douglas Lukins

University of Kentucky College of Medicine

\section{Rita Colwell}

CosmosID, INC

Ai-Ling Lin

University of Missouri-St Louis

Keith Pennypacker ( $\square$ keith.pennypacker@uky.edu )

University of Kentucky https://orcid.org/0000-0002-4618-0903

Research

Keywords: stroke, microbiome, inflammation, imaging

Posted Date: November 8th, 2021

DOI: https://doi.org/10.21203/rs.3.rs-1044260/v1

License: (c) (1) This work is licensed under a Creative Commons Attribution 4.0 International License.

Read Full License 


\section{Abstract}

7 Background: An imbalanced gut microbial community, or dysbiosis, has been shown to occur

\section{Gut microbial dysbiosis is correlated with stroke severity markers in aged rats following stroke}

\author{
Tyler C. Hammond, ${ }^{1,2}$ Sarah Messmer, ${ }^{3,4}$ Jacque A. Frank, ${ }^{3,4}$ Doug Lukins, ${ }^{5}$ Rita Colwell, ${ }^{6}$ \\ Ai-Ling Lin, ${ }^{1,7^{*}}$ and Keith R. Pennypacker ${ }^{3,4^{*}}$
}

following stroke. It is possible that this dysbiosis negatively impacts stroke recovery and

rehabilitation. Species level resolution measurements of the gut microbiome following stroke are needed to develop and test precision interventions such as probiotic or fecal microbiota transplant therapies that target the gut microbiome following stroke. Previous studies have used $16 \mathrm{~S}$ rRNA amplicon sequencing in young male mice to obtain broad profiling of the gut microbiome at the genus level following stroke, but further investigations will be needed with whole genome shotgun sequencing in aged rats of both sexes to obtain species level resolution in a model which will better translate to the demographics of human stroke patients.

Results: 39 aged male and female rats underwent middle cerebral artery occlusion. Fecal samples were collected before stroke and three days post stroke to measure gut microbiome. Machine learning was used to identify the top ranked bacteria which were changed following stroke. MRI imaging was used to obtain infarct and edema size and cerebral blood flow (CBF). ELISA was used to obtain inflammatory markers.

Dysbiosis was demonstrated by an increase in pathogenic bacteria such as Butyricimonas virosa (15.52 fold change, $\mathrm{p}<0.0001$ ), Bacteroides vulgatus ( 7.36 fold change, $\mathrm{p}<0.0001$ ), and Escherichia coli (47.67 fold change, $\mathrm{p}<0.0001$ ). These bacteria were positively associated with infarct and edema size and with the inflammatory markers Cc119, Ccl24, IL17a, IL3, and complement $\mathrm{C} 5$; they were negatively correlated with CBF. Conversely, beneficial bacteria such as Ruminococcus flavefaciens ( 0.14 fold change, $\mathrm{p}<0.0001$ ), Akkermansia muciniphila ( 0.78 fold change, $\mathrm{p}<0.0001$ ), and Lactobacillus murinus ( 0.40 fold change, $\mathrm{p}<0.0001$ ) were decreased following stroke and associated with all the previous parameters in the opposite 
29 direction of the pathogenic species. There were not significant microbiome differences between the sexes.

31 Conclusion: The species level resolution measurements found here can be used as a foundation

32 to develop and test precision interventions targeting the gut microbiome following stroke.

33 Probiotics that include Ruminococcus flavefaciens, Akkermansia muciniphila, and 34 Lactobacillus murinus should be developed to target the deficit following stroke to measure 35 the impact on stroke severity.

37 Author affiliations:

381 Sanders-Brown Center on Aging, University of Kentucky, Lexington, KY 40536 USA

392 Department of Neuroscience, University of Kentucky College of Medicine, Lexington, KY 4040536 USA

413 The Center for Advanced Translational Stroke Science, University of Kentucky, Lexington, 42 KY 40536 USA

434 Department of Neurology, University of Kentucky College of Medicine, Lexington, KY 4440536 USA

455 Department of Radiology, University of Kentucky College of Medicine, Lexington, KY 4640536 USA

476 CosmosID, Inc., Rockville, MD 20850 USA

487 Department of Radiology, Division of Biological Sciences and Institute for Data Science \& 49 Informatics, University of Missouri, Columbia, MO 65211

50 *The authors have equal contribution to the study.

52 Correspondence to:

53 Keith R. Pennypacker, $\mathrm{PhD}$

54 BBSRB, Office B457, University of Kentucky, Lexington, KY 40536

$55 \quad$ Keith.pennypacker@uky.edu

56

57

61 Running title: Gut bacteria tied with stroke severity

62 Keywords: stroke, microbiome, inflammation, imaging 


\section{Background}

64 Over 795,000 people suffer a stroke every year in the United States alone ${ }^{1}$. Recent advances in 65 acute stroke therapies have lowered stroke mortality, but survivors are often left severely 66 impaired ${ }^{2}$. Rehabilitation therapies are beneficial at inducing neuroplasticity to overcome these 67 impairments, but over $40 \%$ of stroke survivors are left with moderate to severe disabilities that markedly reduce quality of life ${ }^{3}$. Novel multimodal approaches are needed to promote plasticity and sensorimotor function through a combination of current rehabilitation therapies with other treatments designed to foster neuroplasticity.

Accumulating evidence suggests that gut microbes modulate brain plasticity via the bidirectional gut-brain axis and may play a role in stroke rehabilitation ${ }^{4}$. A severely imbalanced microbial community, or dysbiosis, has been shown to occur following stroke, causing a systemic flood of neuro- and immunomodulatory substances due to increased gut permeability and decreased gut motility ${ }^{5}$. These substances can impact neuroinflammation as commensal bacteria invade the bloodstream and as intestinal lymphocytes migrate from gut-associated lymphoid tissue to the brain ${ }^{6}$. Fecal microbiota transplant has been shown to normalize brain lesion-induced dysbiosis and to improve stroke outcome in mice ${ }^{6}$. The microbiome is modifiable as it is influenced by environmental factors such as diet and exercise and could potentially be a therapeutic target in stroke rehabilitation through nutritional and pharmacological interventions and physical therapy ${ }^{7,8}$. To our knowledge, no studies have measured the species level resolution necessary to develop precision interventions such as probiotics or fecal microbiota transplants that target the gut microbiota following stroke. Furthermore, no microbiome studies have been performed on aged rats of both sexes, which are better matched to the demographics of human stroke patient than the young male mice used in most studies. The microbiome changes found in this study need to be examined and correlated with clinical imaging markers of stroke and inflammatory markers to understand better whether the microbiome could be a therapeutic target in stroke rehabilitation.

Here we identify the gut-brain axis changes that occur following stroke in aged rats using high resolution whole genome shotgun sequencing and correlate them with clinical imaging markers of stroke including MRI-based infarct size, edema size, and cerebral blood flow (CBF) as well as inflammatory markers. We found that microbial communities are disrupted in an aged rat population following stroke, showing significantly different beta diversity, increased alpha diversity, and changes in the relative abundance of 5 of the 6 major phyla found in the gut. 
95 Changes in thirteen bacterial species as detected by machine learning were highly associated with stroke and changes in these species were also associated with increased infarct and edema size and decreased CBF. Changes in the microbiome due to stroke were also associated with increases in 49 inflammatory markers.

\section{Materials and methods}

\section{Ethics approval and animals}

101 Aged male and female rats (18-month-old Sprague-Dawley rats (ENVIGO, Indianapolis, IN)

102 were used for all procedures. The aged female rats on average weighed between $245 \mathrm{~g}$ and $425 \mathrm{~g}$, 103 and aged male rats approximately weighed between $505 \mathrm{~g}$ and $705 \mathrm{~g}$. The study was conducted 104 in accordance with the National Institutes of Health Guide for the Care and Use of Laboratory 105 Animals and study protocols were approved by University of Kentucky's (UK) Institutional 106 Animal Care and Use Committee. Animals were housed in a climate-controlled room on a 12$107 \mathrm{hr}$ light and dark cycle $(0700-1,900)$ with access to food and water. Per Division of Laboratory 108 Animal Resources (DLAR) cage requirements at UK's vivarium facility, the animals can be 109 paired in one cage if the animal weight is under 650 grams. We typically house two animals 110 (males or females) per cage upon arrival to DLAR. Once the rats are over 650 grams, they are 111 then split into a separate cage by themselves. Fecal samples were collected for all animals at 11224 hours before surgery and 72 hours post-surgery and for 4 animals at 30 days post-surgery.

113 The rats underwent MRI at 72 hours to measure infarct and edema volumes and CBF then 114 euthanized.

\section{Middle cerebral artery occlusion}

11622 of the rats received a permanent Middle Cerebral Artery Occlusion (p-MCAO) and 17 of

117 the rats received a 5-hour transient Middle Cerebral Artery Occlusion (5t-MCAO). All animals 118 were induced with oxygen containing 5\% isoflurane, then shaved, prepped with Hibiclens 
119 (chlorohexidine scrub) prior to $70 \% \mathrm{EtOH}$ and then a betadine solution. Maintenance isoflurane 120 was maintained at $2.5 \%$ in $\mathrm{O} 2$ was delivered via a nosecone placed in line with the binner 121 tubeQ (gas delivery tube) of the anesthesia circuit. Under bnear sterileQ conditions and with 122 the use of a Zeiss operating microscope (Carl Zeiss AG, Gottingen, Germany) at 4 to 25 123 magnification, the procedure was performed. First, the skin was opened with a midline vertical 124 incision, and the underlying submandibular gland bluntly dissected in the midline to produce 125 left and right lobes, which were retracted laterally. Division of the omohyoid muscle, then 126 dissection medial to the right sternocleidomastoid ( $\mathrm{SCM}$ ) muscle was used to expose the 127 common carotid artery (CCA), which was separated from the vagus nerve. Elastic hooks (Lone 128 Star Medical Products, Houston, TX, USA) tethered to metal stays on the customized surgery 129 table were used to retract the skin and the SCM muscle. In the p-MCAO, a hand-held 130 electrocautery (Aaron Medical, St. Petersburg, FL, USA) is used to cauterize the superior 131 thyroid artery (STA), a collateral off the ECA, and the occipital artery (OA), a collateral off 132 the ICA. Two 5-0 silk sutures (Surgical Specialties, Reading, PA, USA) were used to ligate the 133 external carotid artery (ECA) as distal as possible to the ECA/ICA bifurcation, and a second 134 tie that was applied just proximal to the first, leaving enough space in between the two ties to cut the artery with micro scissors. At this point, blunt dissection was used to isolate the internal carotid artery (ICA) and its collateral, the pterygopalatine artery. Next, microvascular aneurysm clips (Mizuho, Beverly, MA, USA) were applied to the CCA and the ICA. A 5-0

138 PDS II monofilament embolus (Ethicon, Cornelia, GA, USA), was introduced into an 139 arteriotomy hole-produced with a 26-gauge hypodermic needle-in the reflected ECA stump 140 and fed distally into the ICA. At this time, a collar suture at the base of the ECA stump was 141 tightened around the embolus, and the ICA clamp was removed. The embolus was advanced $14220 \mathrm{~mm}$ from the carotid bifurcation, with care taken to avoid entrance into the pterygopalatine 143 artery. 
144 For the transient occlusion, the same steps were done as stated with the pMCAO, with the exception that Doccol Corporation silicone rubber-coated monofilaments were used for the occlusion of the middle cerebral artery (MCA). Multiple sized Doccol monofilaments are used

147 in the MCAO surgery depending on the sex and weight of the rat. Two 18-inch length of 5-0

148 silk suture were used for the ligation of the external carotid artery (ECA) to secure the ECA 149 stump, and the entry point of the monofilament into the ECA/ICA bifurcation. The third 5-0 150 silk suture was used to secure the monofilament within the ECA. A micro-serrefines arterial 151 clamp (FST, Fine Science Tools, \#18055-01) was used to occlude the internal carotid artery 152 (ICA) and common carotid artery (CCA) prior to advancement of the monofilament into the 153 MCA. After 5 hours, the embolus was gently removed and the collar suture at the base of the 154 ECA stump tightened. The skin was closed with 3-0 nylon suture (Ethicon, Cornelia, GA, 155 USA), anesthesia discontinued, and the animal allowed to recover. Animals used for control underwent a neck dissection and coagulation of the external carotid artery, but no manipulation

157 or occlusion of the common or internal carotid arteries.

158 Post-surgical fluid management and pain control

159 Immediately post-operatively the animals received $2 \mathrm{ml}$ of sterile saline $(0.9 \%)$ subcutaneous.

160 An additional $1 \mathrm{ml}$ of saline was given if extra blood loss occurred during surgery. The animals were injected with sterile filtered PBS pH 7.4 at 6 (for the p-MCAO), 24, 48, and 72 hours post-MCAO. The animals were weighed every morning post-MCAO to determine dehydration. Hydration status was checked by pinching up or "tenting" the skin over the nape of the neck.

164 The skin should immediately relax into its normal position. If the skin remains tented longer 165 than normal, the rat was deemed dehydrated, and saline was given. Per DLAR guidelines, rats can receive up to $10 \mathrm{ml}$ at a time and no more than $2 \mathrm{ml}$ at any one location per $6 \mathrm{hr}$. If warranted, additional saline (1-2 $\mathrm{ml})$ will be given in addition to $6,24,48$, and $72 \mathrm{hr}$. Also, we 
added an additional water bottle in each cage to allow more avail- ability to free water for the rats to consume and moistened food was provided on the bottom of the cage to encourage feeding and additional water intake. Post-surgical pain control was managed with carprofen,

171 which is based on weight of the animal. Animal weights are taken prior to surgery (pMCAO) 172 and daily until animals are euthanized at $72 \mathrm{hr}$. (post MRI). The animals received a dosage of 173 carprofen $5 \mathrm{mg} / \mathrm{kg}$ prior to surgery and every $24 \mathrm{hr}$. for three days post-pMCAO until $72 \mathrm{hr}$. when they were euthanized (post MRI). Termination of survival criteria include that all animals were weighed and monitored, especially for dehydration and pain, each morning post surgery. This includes specific attention to the animal as a whole, as well as incision sights. If symptoms

177 such as pain, fatigue, loss of energy, excess energy, ruffled hair coat, reluctance to move, failure 178 to groom or feed, hypoactivity, hyperactivity, restlessness, self-trauma, aggressiveness, ataxia, 179 pale mucous membranes, cyanosis, rapid, shallow and/or labored breathing, cachexia, 180 porphyria, soiled anogenital area, inactivity, failure to respond to stimuli, lack of 181 inquisitiveness, vocalization, and/or hunched posture were observed, the research team 182 obtained advice from the vivarium veterinary staff on how best to intervene to alleviate 183 discomfort; if that was not possible the animal was euthanatized. Additional checks were made 184 in the afternoon if there was any rat of concern. The animals were removed from the study if 185 adverse signs persisted despite carprofen and treatment past $24 \mathrm{hr}$. If the signs fail to resolve, 186 the vivarium veterinarian was consulted and decided the time course when such animals were 187 euthanized. Additionally, weight loss greater than 20\% (emaciated appearance, rapid weight 188 loss over two days) was considered an endpoint. Rapid weight loss was considered greater than $189 \quad 10 \%$ a day for two days.

\section{Microbiome Sequencing}


191 Fecal samples were collected for all animals at 24 hours before surgery and 72 hours postsurgery and for 4 animals at 30 days post-surgery. Genomic DNA were extracted from 0.25 grams of stool using ZymoBIOMICS ${ }^{\text {TM }}$ DNA Mini Kit and shipped to CosmosID for DNA quantification using fluorometer Qubit 3.0. Libraries were constructed and the PCR products were purified using 1.0X speed beads and eluted in $15 \mu \mathrm{L}$ of nuclease-free water and quantified by PicoGreen fluorometric assay (100X final dilution). The libraries were pooled and loaded onto a high sensitivity chip run on the Caliper LabChipGX (Perkin Elmer, Waltham, MA) for size estimation and sequenced using Illumina NextSeq/HiSeq platform. Unassembled sequencing reads were analyzed by CosmosID bioinformatics platform (CosmosID Inc.,

200 Rockville, MD) ${ }^{9-12}$ for microbiome analysis. Heatmaps, stacked bar graphs, and Principal

201 Component Analysis (PCA) plots were generated to visualize the diversity and abundance of each microbial taxa. Alpha- and beta-diversity were calculated to determine the number of species present in a cohort and diversity similarities between groups.

\section{Magnetic resonance imaging}

MRI images were acquired on a 7T Bruker Clinscan horizontal bore system $(7.0 \mathrm{~T}, 30 \mathrm{~cm}, 300$ $\mathrm{Hz})$ equipped with a triple-axis gradient system $(630 \mathrm{mT} / \mathrm{m}$ and $6,300 \mathrm{~T} \mathrm{~m}-1 \mathrm{~s}-1)$ with a closed cycle. PCASL (pseudo conintous arterial spin labelling) images were acquired coronally to determine CBF with a fat saturated, double refocused echo planar sequence: TR $4000 \mathrm{~ms}$, TE $26 \mathrm{~ms}$, Matrix 74 x 56, FOV $26 \mathrm{~mm}$ x $19.7 \mathrm{~mm}$, Slice $1.2 \mathrm{~mm}$, Slices 6, 120 Tagged-Untagged

210 Pairs, $10 \mathrm{M}_{0}$ Images, Tagging Plane Offset 12mm, Bolus duration 1.86sec, Post Labeling Delay

2110 sec, and Acquisition Time of $10 \mathrm{~min}$. T2 weighted images were acquired coronally with a

212 RARE sequence: TR 6000 ms, TE 29 ms, Turbo Factor 5, Matrix 190 x 190, FOV 240 mm x

$213240 \mathrm{~mm}$, Slice $0.4 \mathrm{~mm}$, Slices 44, and Acquisition Time of $9 \mathrm{~min}$. Male rats were anesthetized

214 with an average of $2.25 \%$ isoflurane in oxygen, while female rats were anesthetized with an 
215 average of $1.75 \%$ isoflurane in oxygen using an MRI compatible CWE Inc. equipment

216 (Ardmore, PA). They were held in place on a Bruker scanning bed with a tooth bar, ear bars,

217 and tape. Body temperature, heart rate, and respiratory rate were continuously monitored

218 throughout the MRI scans (SA Instruments, Inc., Stony Brook, NY). The animal's body

219 temperatures were maintained at $37^{\circ} \mathrm{C}$ with a water heating system built into the scanning bed.

220 The scanning procedure took approximately 40-60 mins. per animal.

221 The MR images were analyzed by a blinded neuroradiologist who visually identified infarct

222 volume and edema volume. These volumes were counted, and this number was normalized to

223 the number of images counted to provide a per section count. The volume of brain parenchyma

224 demonstrating infarct volume visibly affected was calculated by manual segmentation using 225 ITK-SNAP software (www.itksnap.org, version 3.6) ${ }^{13}$. The volume of brain parenchyma

226 visibly affected by T2 hyperintensity (edema volume) was calculated in a similar fashion. The 227 data are given as absolute volume in cubic millimeters. The calculation was based on all slices 228 from each MR sequence. Cerebral perfusion values of the area of lesion within the ipsilateral 229 hemisphere, and the equivalent region within the contralateral hemisphere were generated 230 using the quantification as previously described. ${ }^{14,15}$

\section{Biochemical analysis}

233 In following STAIR guidelines, clinically relevant biomarkers were determined in our aged 234 male and female rats ${ }^{16}$. Blood was taken from the jugular vein at three different time points: immediately prior to MCAO surgery and 5 mins after reperfusion of the MCA in the pMCAO, and 5 hours post MCAO procedure in the 5t-MCAO. Blood was immediately placed on ice and centrifuged at $2000 \mathrm{~g}$ for 15 minutes. Plasma was extracted and stored separately, both pellet and plasma were frozen at $-80^{\circ} \mathrm{C}$ for later analysis. RNA extraction and Amplification followed 
239 the methods of Martha et.al $2020^{17}$. Briefly, total RNA was extracted from the pellet portion

240 via a Nucleospin Blood Kit (Macherey-Nagel, Düren, Germany), RNA quantity was estimated

241 using a Qubit 4 Fluorometer (Thermo-Fisher; Waltham, MA), cDNA was synthesized using a

$242 \mathrm{RT}^{2}$ PreAMP cDNA synthesis Kit from Qiagen and expression of 84 genes were measured

243 using an ABI StepOne Plus (Germantown, MD) and a $\mathrm{RT}^{2}$ Profiler Rat Chemokine and

244 Receptor Array from Qiagen. Delta Delta CT was calculated using the fold change of the gene 245 expression measurement from pre to 3-day.

\section{Statistical analysis}

247 Descriptive microbiome analyses were performed with CosmosID bioinformatics software to 248 generate alpha diversity, beta diversity, and relative abundance data. Alpha diversities amongst 249 groups were compared using Wilcoxon Rank Sum test. Beta diversities amongst groups were compared using PermANOVA. Relative abundance data was compared to measures of stroke severity as determined by imaging (infarct size, edema size, CBF) using general linear models within the MaAsLin $2 \mathrm{R}_{\text {package }}{ }^{18}$. Random forest was used to determine top bacterial species that were changed following stroke using the randomForest $\mathrm{R}$ package ${ }^{19}$. All imaging variables

254 in the study were transformed to meet assumptions of normality. The transformation procedures began with Shapiro-Wilks and for measures with $\mathrm{p}<0.05$, the variables were square root transformed. A p-value of 0.05 was set a priori to determine statistical significance.

\section{Results}

258 We analyzed all rats before and after middle cerebral artery occlusion and considered sex, 259 surgery type, and treatment with LIF or PBS in the analysis. We administered a leukemia 260 inhibitory factor (LIF) treatment on half of the rats based on previous work suggesting that LIF 261 is an anti-inflammatory that regulates the immune/inflammatory response to stroke ${ }^{20}$. The rats had an average of $96.50 \mathrm{~mm}^{3}$ infarct size, $131.0 \mathrm{~mm}^{3}$ edema size, and $1.31 \mathrm{ml} / \mathrm{g} / \mathrm{min}$ CBF from 
a permanent occlusion and $31.46 \mathrm{~mm}^{3}$ infarct size, $102.1 \mathrm{~mm}^{3}$ edema size, and $2.16 \mathrm{ml} / \mathrm{g} / \mathrm{min}$

$\mathrm{CBF}$ from a transient occlusion. Infarct and edema volumes were not significantly different between sex, treatment group, or occlusion type. No significant difference in CBF was detected between sex or treatment, but, as expected, a significant difference occurred between permanent and transient occlusion in CBF (Fig. 1).

\section{The aged rat gut microbiome is disrupted following stroke}

We performed an analysis on the gut microbial communities of the aged rats before and after stroke. Comparing the alpha diversity before and after stroke, we found that richness and evenness increased from 3.818 on the Shannon diversity index ${ }^{21}$ to 4.178 (Fig. 2A). There were no differences in the change of alpha diversity between sex, treatment, or occlusion type. Comparing the beta diversity before and after stroke, we found that the microbial communities were significantly different between baseline and stroke $(p=0.0001)$, but no significant microbial community differences were detected based on sex, treatment, or occlusion type. (Fig. 2B and Supplementary Table 1).

We investigated specific differences in the relative abundance of the major bacterial phyla in the gut (Fig. 3). We found increases in proteobacteria and Bacteroidetes and decreases in firmicutes, verrucomicrobia, and actinobacteria following stroke (Supplementary Table 2A). This translates to a sharp decrease in the firmicutes to bacteroidetes ratio. Using linear regression, the major bacterial phyla predict infarct size with an $R^{2}=0.3866$ and edema size with an $R^{2}=0.6022$ (Supplementary Table 2B).

\section{The top 13 disrupted bacterial species following stroke}

We investigated specific differences in the relative abundance of the major bacterial species in the gut. There was a total of 29 species increased and 23 species decreased following stroke (Table 1). Supplementary Table 3 gives a detailed description of all the taxa that were increased (red) or decreased (green) following stroke. Using random forest machine learning classification, we found the most important bacterial species that predict stroke verse baseline with an $85.14 \%$ accuracy. They include an increase in Butyricimonas virosa, Bacteroides vulgatus, Escherichia coli, Bacteroides uniformis, Bacteroides dorei, Parabacteroides distasonis, and Alistipes indistinctus and a decrease in Ruminococcus flavefaciens, Akkermansia muciniphila, Ruminococcus_u_s, [Clostridium] clostridioforme, Lactobacillus murinus, and Lachnospiraceae bacterium 3-1. Using linear regression with backwards 
elimination (Table 2), we found that increases in Ruminococcus_u_s and Alistipes indistinctus and decreases in Lachnospiraceae bacterium 3-1 predict infarct volume with an $R^{2}=0.4433$. Increases in Butyricinomas virosa, Bacteroides uniformis, and Ruminococcus_u_s and decreases in Ruminococcus flavefaciens predict edema with an $R^{2}=0.6230$. Finally, decreases in Alistipes indistinctus predict $\mathrm{CBF}$ with an $R^{2}=0.1825$.

299 We investigated potential interactions between bacterial species in predicting infarct size, 300 edema size, and CBF (Supplementary Table 4). Using a feasible solution algorithm (FSA) 301 for finding interactions, we found that decreases in Lachnospiraceae bacterium A2 and Lactobacillus murinus predict infarct size, but a combination of the two predicts a dramatic increase in the prediction value with an $R^{2}=0.6206$. Decreases in Lachnospiraceae bacterium A4 and Lactobacillus murinus predict edema size, but a combination of the two have stronger predictive ability with an $R^{2}=0.6454$. Decreases in Adlercreutzia equolifaciens and Desulfovibrio desulfuricans predict $\mathrm{CBF}$, but again, a combination of the two has a stronger prediction with an $R^{2}=0.8093$.

\section{Bacterial community disruptions following stroke are correlated}

\section{with stroke severity markers}

310 We investigated the correlation of all the bacterial species with infarct size and edema size

311 (Table 3). Using the MaAsLin $2 \mathrm{R}$ package $^{18}$, which automatically normalizes and transforms

312 all variables in preparation for linear regression, we correlated metagenomic sequencing with

313 imaging variables of stroke severity. Twenty-seven bacterial species were positively correlated

314 and 19 negatively correlated with infarct volume. Thirty species were positively correlated,

315 and 31 species were negatively correlated with edema volume. No species were correlated with 316 CBF.

317 Bacterial community disruptions following stroke are correlated 318 with rises in inflammatory markers

319 We investigated the association of inflammatory markers with gut microbiome changes (Table

320 4). Using an Rt2 PCR array ${ }^{22}$ to test the difference between inflammatory genes expressed before and after stroke in a subsample of the rats, we found all the markers that were associated with the changes in gut microbiome. There were 22 bacterial species changed with stroke that 
were also correlated with changes in inflammatory markers. There were 49 total inflammatory markers that were increased in association with bacterial changes (Supplementary Table 5).

\section{Discussion}

326 To our knowledge, we are the first to report on the gut microbial changes with species level 327 resolution in aged male and female rats and to correlate these changes with clinical MRI 328 imaging markers of stroke and inflammatory markers. Following stroke, we found that alpha diversity significantly increased, beta diversity significantly changed, and 5 of the 6 major bacterial phyla were altered. Using machine learning, the top 13 bacterial species that predict whether a sample came from the baseline or post-stroke time point. These bacterial species had independent significant correlations with infarct size, edema size, and CBF. We also identified several species whose interactions with one another were significant in correlating with stroke imaging outcomes. Finally, we found 49 inflammatory markers that correlated with the changes in microbiome from stroke. These changes are representative of a shift from beneficial to pathogenic bacterial species following stroke which results in an increased inflammatory response.

338 Figure 4 summarizes the changes in gut microbial communities in response to stroke.

339 Following stroke there is a significant shift in the gut microbiome, with alterations to 52 major

340 bacterial species. These bacterial fluctuations shift the environment to a more inflammatory 341 state that adversely affect injury. The microbial community dysbiosis is likely due to the increased gut permeability and decreased gut motility in addition to the immunodepression caused by the amplified stress response (increased sympathetic nervous system response and

344 hypothalamic-pituitary-adrenal (HPA) axis response) following stroke ${ }^{23}$. Previous groups have reported a decrease in alpha diversity following stroke in a mouse model ${ }^{6}$ and an increase in a human model ${ }^{24}$. Our findings are consistent with others who have seen that microbial communities differ before and after stroke based on measures of beta diversity ${ }^{25}$. We did not find any significant differences in the microbiome between males and females. Some groups have found sex differences in the microbiome that are largely attributed to hormone differences $^{26}$. It is possible that we did not see these differences because the female rats we used are aged and reproductively senescent.

352 We saw increases in proteobacteria following stroke. In previous studies, proteobacteria have 353 been associated with increased cognitive impairment following stroke ${ }^{27}$. Dysbiosis related to 
metabolic disorders, inflammation, and cancer is often related to an increase in proteobacteria $^{28,29}$. This is possibly due to increased oxygen content in the gut following increases in inflammation, providing an optimal environment for these facultative anaerobes ${ }^{30}$.

357 We also saw decreases in firmicutes and increases in bacteroidetes species. Decreased 358 firmicutes have also been associated with Alzheimer's disease ${ }^{31}$. Obesity is often characterized 359 by a significantly increased firmicutes to bacteroidetes $(\mathrm{F} / \mathrm{B}) \mathrm{ratio}^{32}$; interestingly, our study 360 found that stroke has the opposite effect on F/B ratio. Actinobacteria was significantly 361 decreased following stroke. Actinobacteria downregulates inflammation by production of IL3624 and IL-13 33 and is known to have anti-biofilm properties against pathogenic bacteria ${ }^{34}$. It is possible that a decrease in actinobacteria allows other pathogenic bacteria to flourish.

364 Of the bacteria we found that are increased following stroke, many were of the bacteroides species. Bacteroides species have the ability to reduce oxygen levels and breakdown food products to liberate fucose and sialic acid residues from glycoproteins that can be consumed by other microorganisms, including pathogens. Higher bacteroides species are associated with type I diabetes ${ }^{35}$. Bacteroides vulgatus and Bacteroides dorei reduce gut microbial lipopolysaccharide production and inhibit atherosclerosis ${ }^{36}$, but they are also associated with insulin resistance, altered bile acid metabolism, and reduced interleukin-22 secretion $^{37}$. Butyricimonas virosa, Escherichia coli, and Parabacteroides distasonis were also elevated following stroke. An increase of Butyricimonas virosa has also been seen in divers with high occupational exposure to a hyperoxic environment ${ }^{38}$, which is very different from the hypoxic environment of stroke. Escherichia coli is a very common commensal bacteria that has the potential to cause extraintestinal infections based on its genome content and phenotypic traits ${ }^{39}$ and is famous for causing post-stroke infections, especially pneumonia. Parabacteroides distasonis has been shown to alleviate obesity and metabolic dysfunctions via production of succinate and secondary bile acids ${ }^{40}$, which is interesting since stroke is often associated with obesity and metabolic dysfunctions.

Many bacteria which are generally considered beneficial were decreased following stroke including akkermansia, lactobacillus, and ruminococcus species. Akkermansia muciniphila is a mucin-degrading bacterium ${ }^{41}$ that can be increased with fasting ${ }^{42}$ that is known to improve host metabolic functions and immune responses ${ }^{43}$. Lactobacillus murinus can combat inflammaging $^{44}$, and a reduction of $L$. murinus due to high salt consumption has been 
associated with an increase in proinflammatory TH17 cells ${ }^{45}$, which have been correlated with post stroke dysbiosis and secondary injury ${ }^{46}$. Lactobacillus reuteri was also significantly reduced following stroke. A randomized control trial in children showed administration of $L$. reuteri as a probiotic to be useful in treating constipation in children ${ }^{47}$. Constipation is a common morbidity in stroke, and administration of this species could help to alleviate symptoms. Ruminococcus flavefaciens has also been shown to decrease the therapeutic effects of antidepressants, having implications for the treatment of post-stroke depression.

Many of the bacterial changes were associated with increases in inflammatory markers. The major markers that were increased were CCL19, CCL24, IL-17A, IL-3, and complement factor C5. CCL19 is a chemokine that is commonly upregulated as a result of viral infections ${ }^{48}$, and attracts dendritic cells and $\mathrm{T}$ lymphocytes ${ }^{49}$; it promotes thymocyte development, secondary lymphoid organogenesis, high affinity antibody responses, regulatory and memory $\mathrm{T}$ cell function, and lymphocyte egress from tissues organs ${ }^{50,51}$. CCL19 suppresses angiogenesis and can inhibit proliferation, migration, and sprouting responses of tumors ${ }^{52}$. CCL19 has previously been found to be upregulated following stroke after damage to the intestinal epithelium ${ }^{53}$ and has been shown to facilitate T-cell migration to the insult site and microglial activation following stroke ${ }^{54}$. CCL24 plays an important role in pathological processes of skin and lung inflammation and fibrosis ${ }^{55}$ and regulates inflammatory and fibrotic activities through its receptor, CCR $3^{56}$. CCR3 is a mediator of neural cell death ${ }^{57}$. In host defense, IL-17A has been shown to be mostly beneficial against infection caused by extracellular bacteria and fungi ${ }^{58}$ and IL-17A has been shown to be increased following stroke, especially in males ${ }^{59}$. IL3 is strongly associated with brain volume variation and plays pivotal roles in the expansion and maintenance of the neural progenitor pool and the number of surviving neurons ${ }^{60}$; our work has previously identified IL3 increased in the spleen with our aged rat model of stroke ${ }^{20}$. Activation of complement C5 generates the potent anaphylatoxin C5a and leads to pathogen lysis, inflammation, and cell damage ${ }^{61}$. Activated $\mathrm{C} 5$ complement components are a part of the cerebral tissue inflammation following ischemia ${ }^{62}$.

This study lays an important foundation upon which precision interventions can be developed to target the gut microbiome in stroke rehabilitation. Future studies should attempt to manipulate the microbiome to change stroke outcomes. This could be achieved through diet interventions, antibiotic therapy, probiotics, or fecal microbiota transplant. For example, a 
418 future probiotics study should include the use of Ruminococcus flavefaciens, Akkermansia 419 muciniphila, and Lactobacillus murinus as these were deficient in our population. Stroke 420 severity measures from imaging and inflammatory markers could be used as outcomes to 421 compare to the current study. While the present study identified associations of various

422 inflammatory markers with changes in gut microbial composition, it would also be useful to 423 perform mechanistic studies to determine how the microbiota change the expression of these 424 markers and what their downstream effects are. Finally, human studies will be needed to 425 determine whether the microbial changes seen in animals following stroke are similar to the 426 changes seen in animals. Such results can then be used to alter the gut microbiome to favor 427 positive clinical outcomes after stroke.

428

\section{Conclusion}

430 We found that alpha diversity significantly increased following stroke irrespective of sex, treatment, or occlusion type. Beta diversity was also significantly different, with increases in proteobacteria and decreases in the firmicutes to bacteroidetes ratio. Random forest analysis revealed the top 13 species changes as a result of stroke including increases in Butyricimonas virosa and Escherichia coli and decreases in Akkermansia muciniphila and Bacteroides dorei. Correlation analysis revealed that these species changes were associated with increased infarct and edema sizes following stroke. Furthermore, the bacterial changes were associated with increases in inflammatory markers, notably Cc119, Ccl24, IL17a, IL3, and complement C5.

\section{Declarations}

\section{Ethics approval}

440 The study was conducted in accordance with the National Institutes of Health Guide for the

441 Care and Use of Laboratory Animals and study protocols were approved by University of 442 Kentucky's (UK) Institutional Animal Care and Use Committee.

\section{Consent for publication}


$444 \quad$ Not applicable

445 Availability of data and materials

446 All data generated or analyzed during this study are included in this published article

447 Competing interests

448 The authors declare that they have no competing interests

$449 \quad$ Funding

450 This research was supported by NIH/NIA grants RF1AG062480-01S1 to ALL and TRIAD

451 grant training T32AG057461 to TCH. 7T ClinScan small animal MRI scanner of UK was

452 funded by the S10 NIH Shared Instrumentation Program Grant (1S10RR029541-01).

\section{Authors' contributions}

$454 \mathrm{TCH}$ processed the fecal samples, analyzed the data, and prepared the manuscript. SM 455 performed the stroke surgeries. JAF collected the fecal pellets, performed the imaging, and ran 456 the inflammatory analysis. DL interpreted the imaging findings. RC processed the microbiome 457 samples. A-LL and KRP oversaw the design and analysis of all experiments. All authors read 458 and approved the final manuscript.

459 Acknowledgements

$460 \quad$ Not applicable

461

462 Supplementary material

463 Supplementary material is available at Brain online

464 
467 1. Benjamin, E.J., et al. Heart Disease and Stroke Statistics-2017 Update: A Report From the American Heart Association. Circulation 135, e146-e603 (2017).

2. Moy, E., et al. Leading Causes of Death in Nonmetropolitan and Metropolitan AreasUnited States, 1999-2014. Morbidity and mortality weekly report. Surveillance summaries (Washington, D.C. : 2002) 66, 1-8 (2017).

3. Carandang, R., et al. Trends in incidence, lifetime risk, severity, and 30-day mortality of stroke over the past 50 years. Jama 296, 2939-2946 (2006).

4. Leung, K. \& Thuret, S. Gut Microbiota: A Modulator of Brain Plasticity and Cognitive Function in Ageing. Healthcare (Basel, Switzerland) 3, 898-916 (2015).

5. Stanley, D., Moore, R.J. \& Wong, C.H.Y. An insight into intestinal mucosal microbiota disruption after stroke. Scientific reports 8, 568 (2018).

6. Singh, V., et al. Microbiota Dysbiosis Controls the Neuroinflammatory Response after Stroke. The Journal of neuroscience : the official journal of the Society for Neuroscience 36, 7428-7440 (2016).

7. Winek, K., Meisel, A. \& Dirnagl, U. Gut microbiota impact on stroke outcome: Fad or fact? Journal of cerebral blood flow and metabolism : official journal of the International Society of Cerebral Blood Flow and Metabolism 36, 891-898 (2016).

8. Mailing, L.J., Allen, J.M., Buford, T.W., Fields, C.J. \& Woods, J.A. Exercise and the Gut Microbiome: A Review of the Evidence, Potential Mechanisms, and Implications for Human Health. Exerc Sport Sci Rev 47, 75-85 (2019).

9. Ottesen, A., et al. Enrichment dynamics of Listeria monocytogenes and the associated microbiome from naturally contaminated ice cream linked to a listeriosis outbreak. BMC Microbiol 16, 275 (2016).

10. Ponnusamy, D., et al. Cross-talk among flesh-eating Aeromonas hydrophila strains in mixed infection leading to necrotizing fasciitis. Proc Natl Acad Sci U S A 113, 722-727 (2016).

11. Hasan, N.A., et al. Microbial community profiling of human saliva using shotgun metagenomic sequencing. PLoS One 9, e97699 (2014).

12. Lax, S., et al. Longitudinal analysis of microbial interaction between humans and the indoor environment. Science 345, 1048-1052 (2014).

13. Yushkevich, P.A., et al. User-guided 3D active contour segmentation of anatomical structures: significantly improved efficiency and reliability. Neuroimage 31, 1116-1128 (2006).

14. Lin, A.L., et al. APOE genotype-dependent pharmacogenetic responses to rapamycin for preventing Alzheimer's disease. Neurobiol Dis 139, 104834 (2020).

15. Lin, A.L., Zhang, W., Gao, X. \& Watts, L. Caloric restriction increases ketone bodies metabolism and preserves blood flow in aging brain. Neurobiol Aging 36, 2296-2303 (2015).

16. Mehra, M., et al. Preclinical acute ischemic stroke modeling. J Neurointerv Surg 4, 307-313 (2012).

17. Martha, S.R., et al. Expression of Cytokines and Chemokines as Predictors of Stroke Outcomes in Acute Ischemic Stroke. Front Neurol 10, 1391 (2019).

18. Mallick, H., et al. Multivariable Association Discovery in Population-scale Meta-omics Studies. bioRxiv, 2021.2001.2020.427420 (2021).

19. Liaw, A. \& Wiener, M. Classification and Regression by RandomForest. Forest 23(2001). 
20. Davis, S.M., Collier, L.A., Messmer, S.J. \& Pennypacker, K.R. The Poststroke Peripheral Immune Response Is Differentially Regulated by Leukemia Inhibitory Factor in Aged Male and Female Rodents. Oxid Med Cell Longev 2020, 8880244 (2020).

21. Longuet-Higgins, M.S. On the Shannon-Weaver index of diversity, in relation to the distribution of species in bird censuses. Theor Popul Biol 2, 271-289 (1971).

22. Attal, J., Puissant, C. \& Houdebine, L.M. An improvement of a rapid method using Qiagen columns to purify plasmids. Biotechniques 8, 269-271 (1990).

23. Benakis, C., et al. The microbiome-gut-brain axis in acute and chronic brain diseases. Curr Opin Neurobiol 61, 1-9 (2020).

24. Yin, J., et al. Dysbiosis of Gut Microbiota With Reduced Trimethylamine-N-Oxide Level in Patients With Large-Artery Atherosclerotic Stroke or Transient Ischemic Attack. J Am Heart Assoc 4(2015).

25. Park, M.J., et al. Reproductive Senescence and Ischemic Stroke Remodel the Gut Microbiome and Modulate the Effects of Estrogen Treatment in Female Rats. Transl Stroke Res 11, 812-830 (2020).

26. Ahmed, S. \& Spence, J.D. Sex differences in the intestinal microbiome: interactions with risk factors for atherosclerosis and cardiovascular disease. Biol Sex Differ 12, 35 (2021).

27. Ling, Y., et al. Gut Microbiome Signatures Are Biomarkers for Cognitive Impairment in Patients With Ischemic Stroke. Front Aging Neurosci 12, 511562 (2020).

28. Shin, N.R., Whon, T.W. \& Bae, J.W. Proteobacteria: microbial signature of dysbiosis in gut microbiota. Trends Biotechnol 33, 496-503 (2015).

29. Rizzatti, G., Lopetuso, L.R., Gibiino, G., Binda, C. \& Gasbarrini, A. Proteobacteria: A Common Factor in Human Diseases. Biomed Res Int 2017, 9351507 (2017).

30. Rivera-Chavez, F., Lopez, C.A. \& Baumler, A.J. Oxygen as a driver of gut dysbiosis. Free Radic Biol Med 105, 93-101 (2017).

31. Vogt, N.M., et al. Gut microbiome alterations in Alzheimer's disease. Scientific reports 7, 13537 (2017).

32. Magne, F., et al. The Firmicutes/Bacteroidetes Ratio: A Relevant Marker of Gut Dysbiosis in Obese Patients? Nutrients 12(2020).

33. Binda, C., et al. Actinobacteria: A relevant minority for the maintenance of gut homeostasis. Dig Liver Dis 50, 421-428 (2018).

34. Azman, A.S., Mawang, C.I., Khairat, J.E. \& AbuBakar, S. Actinobacteria-a promising natural source of anti-biofilm agents. Int Microbiol 22, 403-409 (2019).

35. Wexler, A.G. \& Goodman, A.L. An insider's perspective: Bacteroides as a window into the microbiome. Nat Microbiol 2, 17026 (2017).

36. Yoshida, N., et al. Bacteroides vulgatus and Bacteroides dorei Reduce Gut Microbial Lipopolysaccharide Production and Inhibit Atherosclerosis. Circulation 138, 24862498 (2018).

37. Qi, X., et al. Gut microbiota-bile acid-interleukin-22 axis orchestrates polycystic ovary syndrome. Nat Med 25, 1225-1233 (2019).

38. Yuan, Y., et al. Changes in the gut microbiota during and after commercial heliumoxygen saturation diving in China. Occup Environ Med 76, 801-807 (2019).

39. Leimbach, A., Hacker, J. \& Dobrindt, U. E. coli as an all-rounder: the thin line between commensalism and pathogenicity. Curr Top Microbiol Immunol 358, 3-32 (2013).

40. Wang, K., et al. Parabacteroides distasonis Alleviates Obesity and Metabolic Dysfunctions via Production of Succinate and Secondary Bile Acids. Cell Rep 26, 222235 e225 (2019). 
41. Derrien, M., Vaughan, E.E., Plugge, C.M. \& de Vos, W.M. Akkermansia muciniphila gen. nov., sp. nov., a human intestinal mucin-degrading bacterium. Int $J$ Syst Evol Microbiol 54, 1469-1476 (2004).

42. Sonoyama, K., et al. Response of gut microbiota to fasting and hibernation in Syrian hamsters. Appl Environ Microbiol 75, 6451-6456 (2009).

43. Zhang, T., Li, Q., Cheng, L., Buch, H. \& Zhang, F. Akkermansia muciniphila is a promising probiotic. Microb Biotechnol 12, 1109-1125 (2019).

44. Pan, F., et al. Predominant gut Lactobacillus murinus strain mediates antiinflammaging effects in calorie-restricted mice. Microbiome 6, 54 (2018).

45. Wilck, N., et al. Salt-responsive gut commensal modulates TH17 axis and disease. Nature 551, 585-589 (2017).

46. Arya, A.K. \& Hu, B. Brain-gut axis after stroke. Brain Circ 4, 165-173 (2018).

47. Kubota, M., et al. Lactobacillus reuteri DSM 17938 and Magnesium Oxide in Children with Functional Chronic Constipation: A Double-Blind and Randomized Clinical Trial. Nutrients 12(2020).

48. Yan, Y., et al. CCL19 and CCR7 Expression, Signaling Pathways, and Adjuvant Functions in Viral Infection and Prevention. Front Cell Dev Biol 7, 212 (2019).

49. Iida, Y., et al. Local injection of CCL19-expressing mesenchymal stem cells augments the therapeutic efficacy of anti-PD-L1 antibody by promoting infiltration of immune cells. J Immunother Cancer 8(2020).

50. Comerford, I., et al. A myriad of functions and complex regulation of the CCR7/CCL19/CCL21 chemokine axis in the adaptive immune system. Cytokine Growth Factor Rev 24, 269-283 (2013).

51. Adachi, K., et al. IL-7 and CCL19 expression in CAR-T cells improves immune cell infiltration and CAR-T cell survival in the tumor. Nat Biotechnol 36, 346-351 (2018).

52. $\mathrm{Xu}, \mathrm{Z}$., et al. CCL19 suppresses angiogenesis through promoting miR-206 and inhibiting Met/ERK/Elk-1/HIF-1alpha/VEGF-A pathway in colorectal cancer. Cell Death Dis 9, 974 (2018).

53. Liu, Y., et al. Ischemic stroke damages the intestinal mucosa and induces alteration of the intestinal lymphocytes and CCL19 mRNA in rats. Neurosci Lett 658, 165-170 (2017).

54. Noor, S. \& Wilson, E.H. Role of C-C chemokine receptor type 7 and its ligands during neuroinflammation. J Neuroinflammation 9, 77 (2012).

55. Mor, A., et al. Blockade of CCL24 with a monoclonal antibody ameliorates experimental dermal and pulmonary fibrosis. Ann Rheum Dis 78, 1260-1268 (2019).

56. Segal-Salto, M., et al. A blocking monoclonal antibody to CCL24 alleviates liver fibrosis and inflammation in experimental models of liver damage. JHEP Rep $\mathbf{2}$, 100064 (2020).

57. Zhang, J., et al. High-Content Genome-Wide RNAi Screen Reveals CCR3 as a Key Mediator of Neuronal Cell Death. eNeuro 3(2016).

58. Chen, K. \& Kolls, J.K. Interluekin-17A (IL17A). Gene 614, 8-14 (2017).

59. El-Hakim, Y., et al. Sex differences in stroke outcome correspond to rapid and severe changes in gut permeability in adult Sprague-Dawley rats. Biol Sex Differ 12, 14 (2021).

60. Luo, X.J., et al. The interleukin 3 gene (IL3) contributes to human brain volume variation by regulating proliferation and survival of neural progenitors. PLoS One 7, e50375 (2012).

61. Jore, M.M., et al. Structural basis for therapeutic inhibition of complement C5. Nat Struct Mol Biol 23, 378-386 (2016).

62. Costa, C., et al. Role of complement component C5 in cerebral ischemia/reperfusion injury. Brain Res 1100, 142-151 (2006). 


\section{Figure legends}

614 Figure 1: Imaging features following stroke

615 Figure 2: Diversity changes following stroke. A) Alpha diversity as measured by the 616 Shannon diversity index detecting species richness and evenness is increased following stroke.

617 There is no difference in change across sex, treatment, or stroke type. B) Beta Diversity as 618 measured by Bray-Curtis method comparing how different samples are

619 Figure 3: Phyla changes as a result of stroke. Relative Abundance shows phyla composition 620 before and after stroke.

621 Figure 4. Summary Figure depicting changes in gut microbial communities in response 622 to stroke.

623

624 
Figures
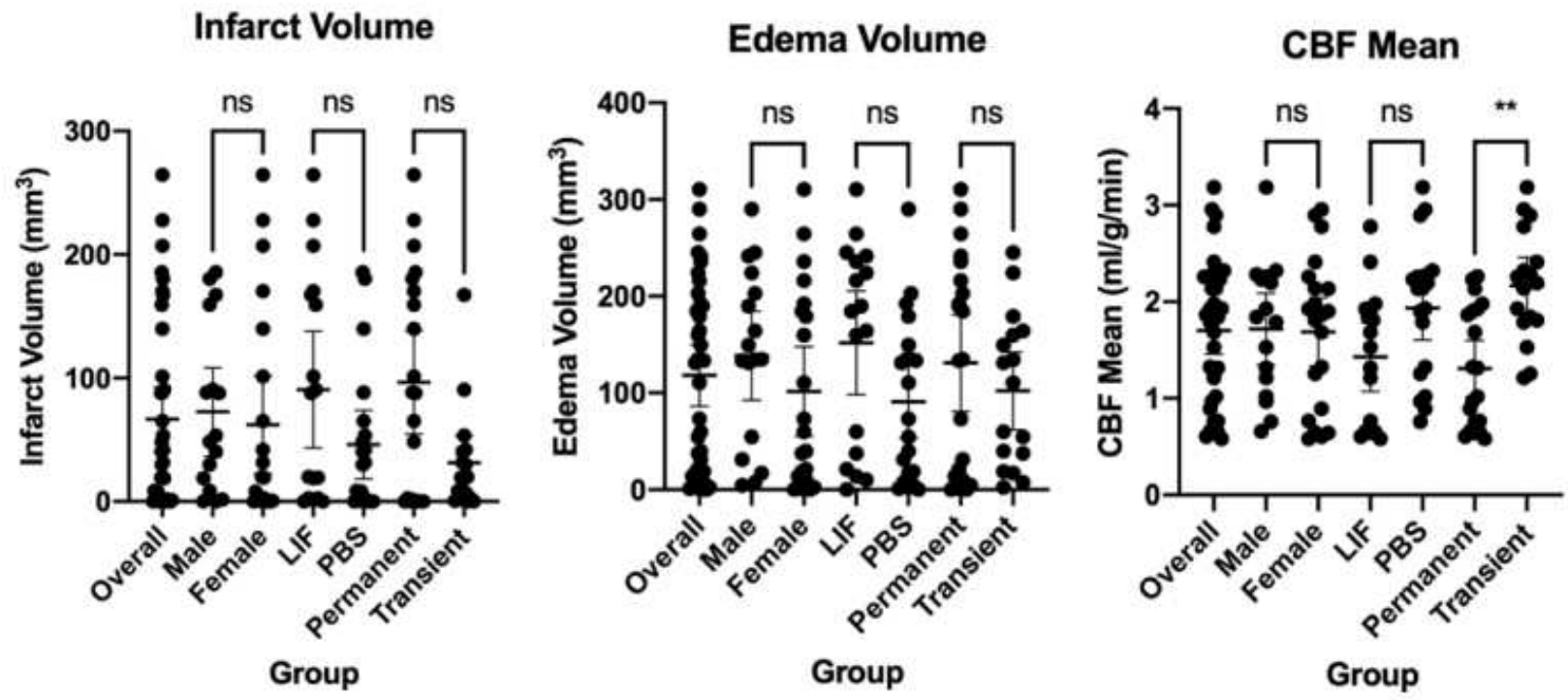

Figure I

Figure 1

Imaging features following stroke 


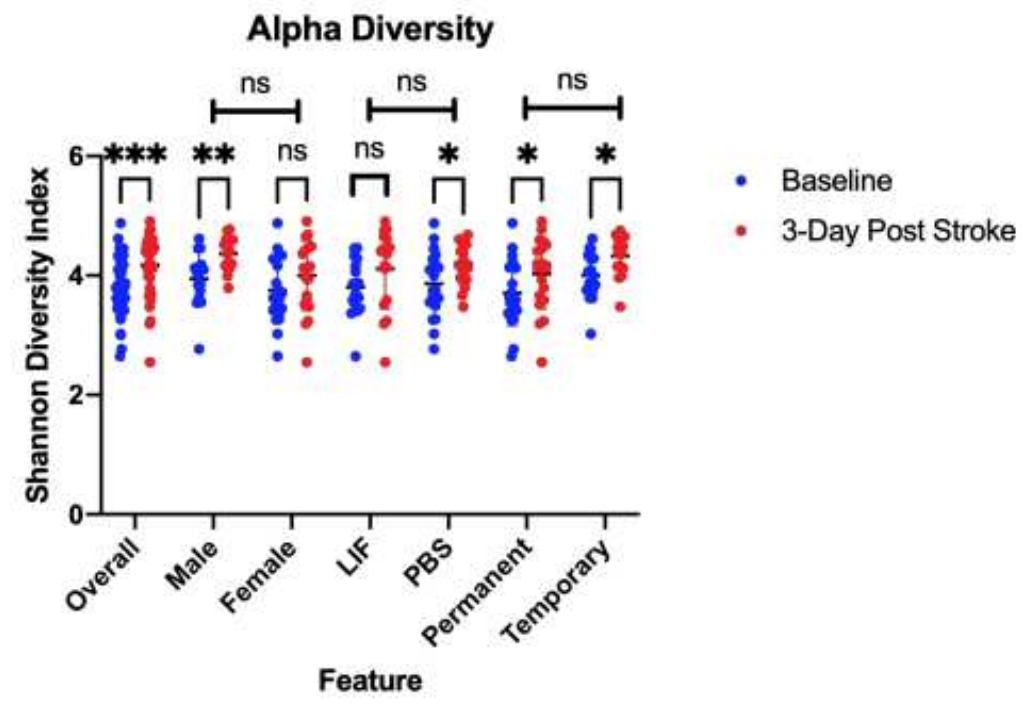

B)

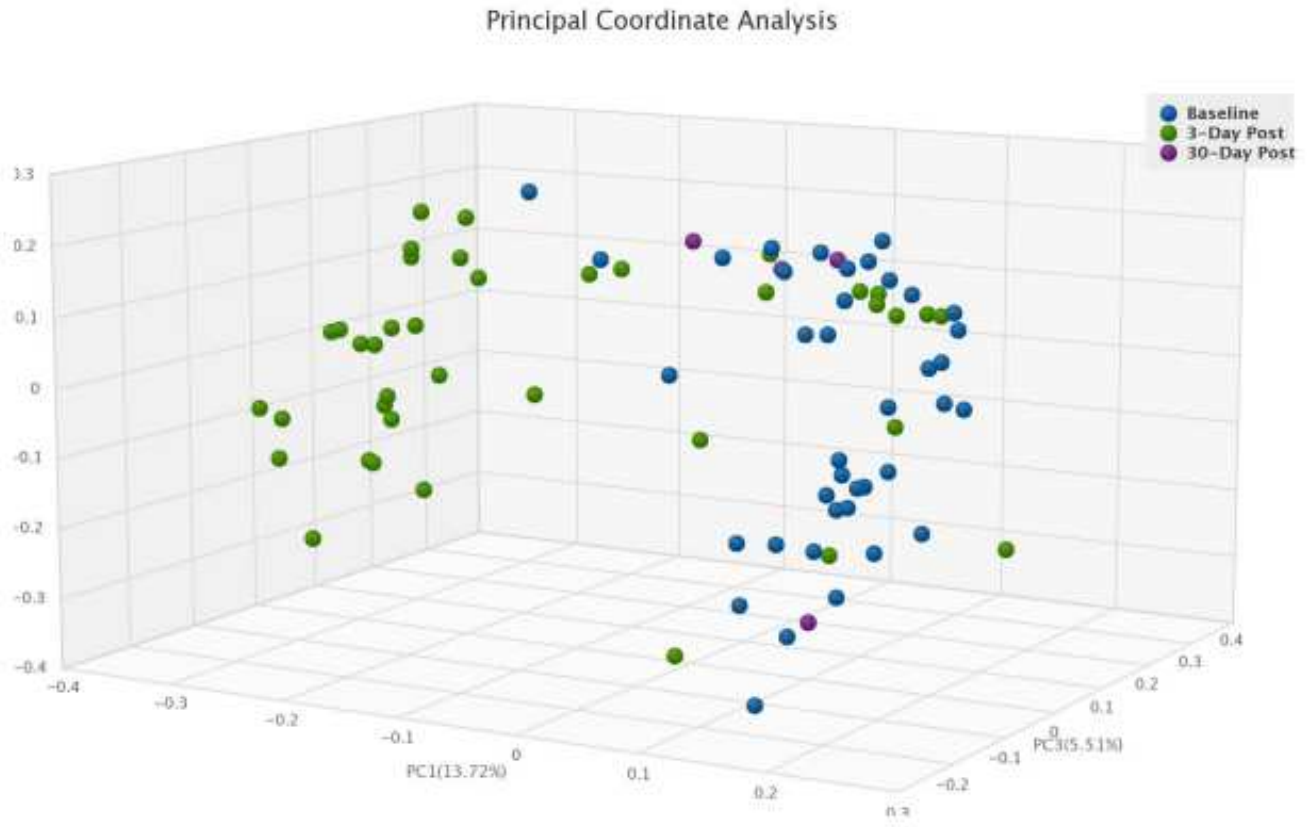

Figure 2

\section{Figure 2}

Diversity changes following stroke. A) Alpha diversity as measured by the Shannon diversity index detecting species richness and evenness is increased following stroke. There is no difference in change across sex, treatment, or stroke type. B) Beta Diversity as measured by Bray-Curtis method comparing how different samples are 


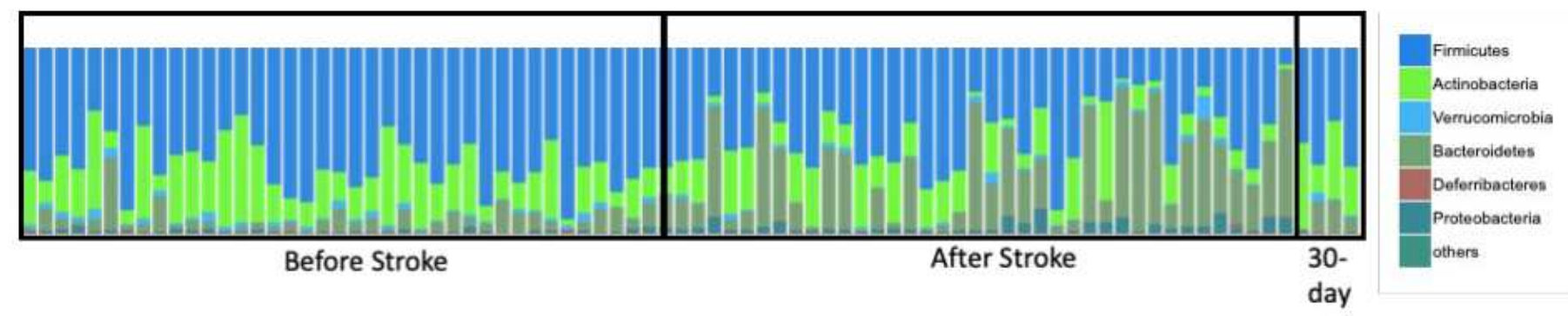

Figure 3

\section{Figure 3}

Phyla changes as a result of stroke. Relative Abundance shows phyla composition before and after stroke. 


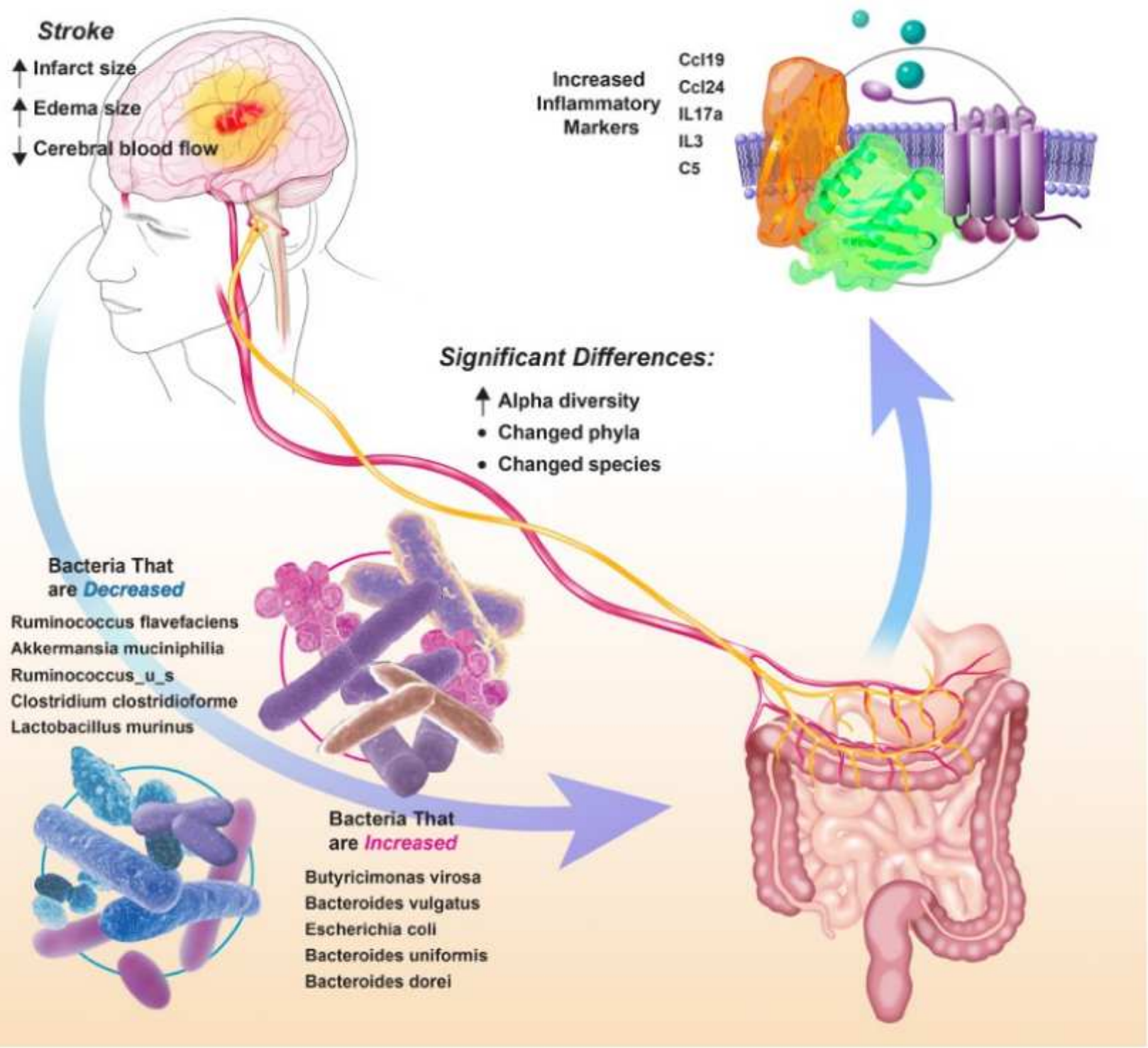

Figure 4

\section{Figure 4}

Summary Figure depicting changes in gut microbial communities in response to stroke.

\section{Supplementary Files}

This is a list of supplementary files associated with this preprint. Click to download.

- RatSupplementalTablesandFigures.pdf 